\title{
Fontan failure and the role of pulmonary vasodilator therapy
}

\author{
Paul Clift ${ }^{1,2}$
}

\begin{abstract}
Background: The Fontan circulation is a palliative procedure for patients born with a single ventricle physiology. The Fontan circulation is associated with significant late morbidity commonly including atrial arrhythmias.

Case presentation: We report a case of an adult Fontan patient who developed protein losing enteropathy (PLE) following persistent atrial arrhythmias. Treatment with a pulmonary vasodilator, sildenafil, resolved the PLE.

Conclusions: The importance of a low pulmonary vascular resistance to maintain wellbeing in the Fontan patient is discussed, as is the role of pulmonary vasodilators.
\end{abstract}

Keywords: Fontan circulation, Pulmonary vasodilators, Pulmonary vascular resistance, Protein losing enteropathy

\section{Background}

It is more than 50 years since Francis Fontan and the Kreutzer brothers performed their initial series of surgical palliation of the single ventricle circulation, now known as the Fontan operation [1,2]. The concept of bypassing the systemic venous return from the systemic ventricle and allowing passive flow to the pulmonary arteries was simple and remarkably effective. Prior to Fontan surgery the life expectancy for patients with a single ventricle circulation was poor and patients rarely survived beyond childhood years, the challenges of profound cyanosis and a volume loaded single ventricle leading to circulatory failure in early life. Subsequent modifications of the procedure have improved outcomes $[3,4]$ and patients routinely survive to adult life, even in the most complex forms of single ventricle circulations [5].

The Fontan circulation is unique and poses many challenges (Fig. 1a and b). Patients, whilst overall well, are prone to developing arrhythmias and exercise

Correspondence: Paul.Clift@uhb.nhs.uk

'Department of Cardiology, Queen Elizabeth Hospital, Birmingham, United Kingdom

${ }^{2}$ Adult Congenital Heart Disease Unit, Queen Elizabeth Hospital, B15 2TH Birmingham, UK

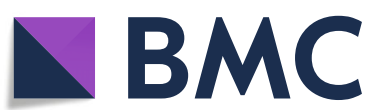

intolerance. Critical to a healthy Fontan circulation is the rapid transit of the systemic venous return through the pulmonary circulation in order to maintain pre-load in the systemic circulation. Therefore, even small increases in the pulmonary vascular resistance may lead to a deterioration in exercise capacity. In the classical atriopulmonary Fontan operation, a rise in pulmonary resistance may lead to increased right atrial chamber pressures and, in combination with multiple surgical scars, predispose to atrial arrhythmia. Such arrhythmias may become life threatening in this population and can lead to rapid clinical deterioration. Moreover, elevation of the systemic venous pressure is an unavoidable feature of the Fontan circulation and is increasingly recognised as a contributory factor to morbidity, including arrhythmia, hepatic fibrosis and the development of protein losing enteropathy (PLE).

We report a case in which pulmonary vasodilator therapy was used successfully to treat PLE in an adult Fontan patient.

\section{Case presentation}

A 28-year-old patient presented with PLE and recurrent persistent atrial arrhythmias. She had been born with 
A

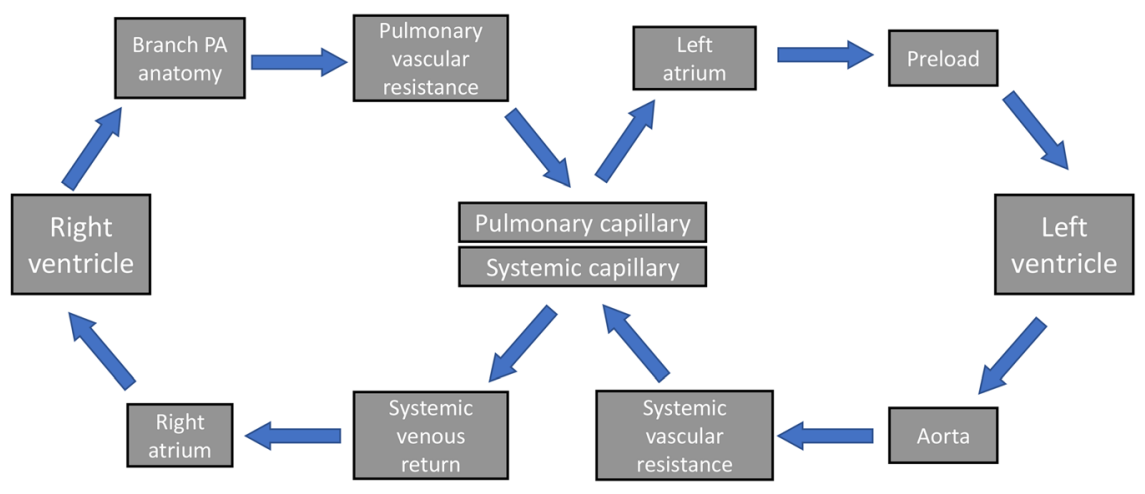

Normal biventricular circulation

B

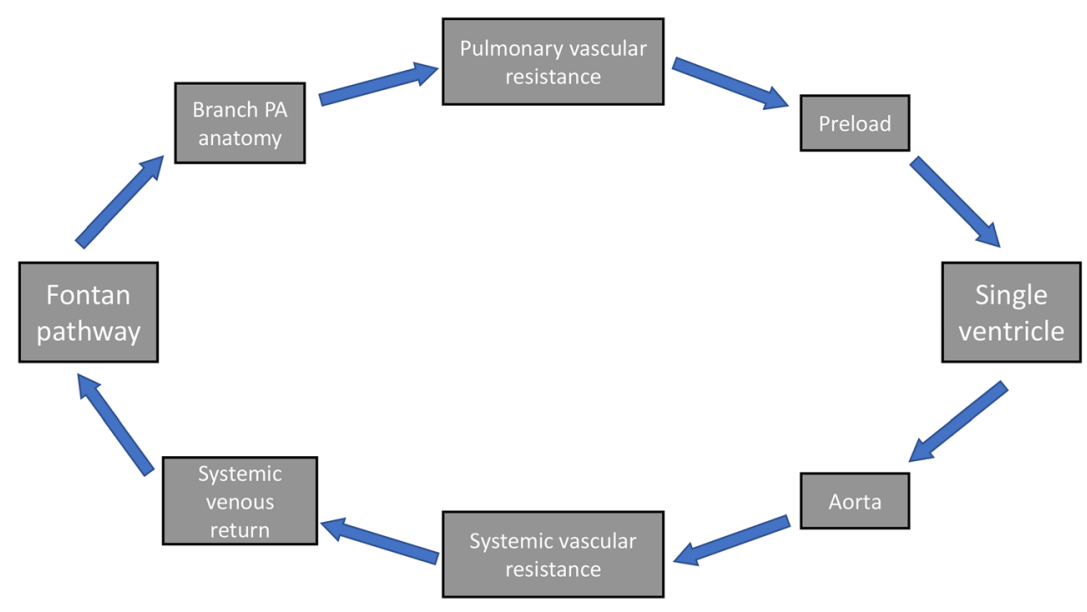

Fontan circulation

Fig. 1 Panel a The normal biventricular circulation. Panel b The Fontan circulation

tricuspid atresia and had undergone initial palliation in infancy with a central systemic arterial shunt (aorta to pulmonary artery anastomosis). She subsequently underwent a single stage atrio-pulmonary Fontan operation at the age of 4 years. Her only long-term medication was oral anticoagulation with warfarin. She maintained good international normalised ratio (INR) control.

Prior to this presentation she had been asymptomatic (World Health Organization Functional Class 1) with a normal (age, sex sized matched to general population) peak oxygen consumption on cardiopulmonary exercise testing. She presented to her local hospital with breathlessness, fatigue and a recent history of intermittent palpitations. She was in a scar-related macro re-entrant atrial tachycardia, and examination demonstrated dependent oedema and ascites. Her albumin was mildly reduced at $29 \mathrm{~g} / \mathrm{l}$. She underwent direct current cardioversion under brief general anaesthesia, and sinus rhythm was restored.

In cases such as this, it is necessary to exclude acute causes of deterioration and triggers for arrhythmia, such as infection (including endocarditis), thyrotoxicosis, valvular heart disease, ventricular dysfunction and thrombosis within the Fontan circulation. Transthoracic echocardiography showed normal systemic left ventricular function and no mitral valve incompetence or left 
ventricular outflow tract obstruction. Thyroid function and inflammatory markers were normal, and there was no clinical evidence of endocarditis. A pregnancy test was negative.

3A transoesophageal echocardiogram (Fig. 2) showed no thrombus within the Fontan circulation, but there was marked spontaneous contrast within the atrio-pulmonary Fontan chamber. There was no pulmonary venous compression and the Fontan pathway was unobstructed.

PLE was confirmed by elevated faecal alpha- 1 antitrypsin levels and a serum albumin level of $29 \mathrm{~g} / \mathrm{l}$. She remained in PLE despite maintaining sinus rhythm, with persistent ascites and peripheral oedema. We therefore elected to perform cardiac catheterisation under local anaesthesia. Mean Fontan chamber pressure was $13 \mathrm{mmHg}$ and there was no gradient on pullback from the pulmonary arteries to the Fontan chamber. The pulmonary capillary wedge pressure was $12 \mathrm{mmHg}$, demonstrating a low transpulmonary gradient, hence, suggesting a low pulmonary vascular resistance.

Despite the haemodynamic findings we elected to commence targeted pulmonary vasodilator therapy with the oral phosphodiesterase inhibitor sildenafil. After tolerating an initial test dose of $12.5 \mathrm{mg}$ we increased this to $25 \mathrm{mg}$ tds. There was rapid resolution of the ascites and oedema, and albumin normalised within 4 weeks.

She remained well under follow up until 7 years later, when she had a reoccurrence of PLE with an albumin of $32 \mathrm{~g} / \mathrm{l}$, dependent oedema and ascites despite being in sinus rhythm. There were no new findings on repeat investigations, with preserved ventricular function, no mitral incompetence and no evidence of Fontan pathway obstruction. We increased her sildenafil to $100 \mathrm{mg}$ tds with full resolution of the PLE within two weeks. She remains well under follow up.

\section{Discussion}

PLE is a poor prognostic sign in patients with a Fontan circulation. Whilst the pathophysiology of the condition is not fully understood, it is postulated that increased systemic venous pressures lead to increased pressure within the thoracic duct, and an increase in gut lymphatic pressure resulting in protein loss in the gastrointestinal tract. Any factor leading to increased systemic venous pressures in the Fontan circulation could lead to the development of PLE. Multiple factors leading to elevation in pulmonary venous pressure such as arrhythmia, atrioventricular valve incompetence or ventricular failure could lead to an increase in pulmonary artery pressure and therefore systemic venous pressure. Similarly pulmonary vascular remodelling with a modest increase in pulmonary vascular resistance or narrowing of any part of the Fontan pathway could do the same, or a combination of any of these factors.

In this case we believe that despite the apparent good haemodynamics, the combination of an arrhythmia and mild pulmonary vascular remodelling may have triggered the onset of PLE and could respond to targeted pulmonary vasodilator therapy. Our practice has been to use a phosphodiesterase $\mathrm{V}$ inhibitor (PDEVi) as first line therapy, due to the rapid onset of action and availability and the lack of evidence base for all therapies at that time. We employed a strategy of increasing dose of sildenafil due to the known potential tachyphylaxis associated with sildenafil and following discussions with the patient, who preferred this strategy.

Given the nature of the Fontan circulation, small changes in pulmonary vascular resistance whilst diminishing transpulmonary blood flow will not necessarily increase the measured pulmonary arterial pressure, due the large capacitance available in the venous circulation.

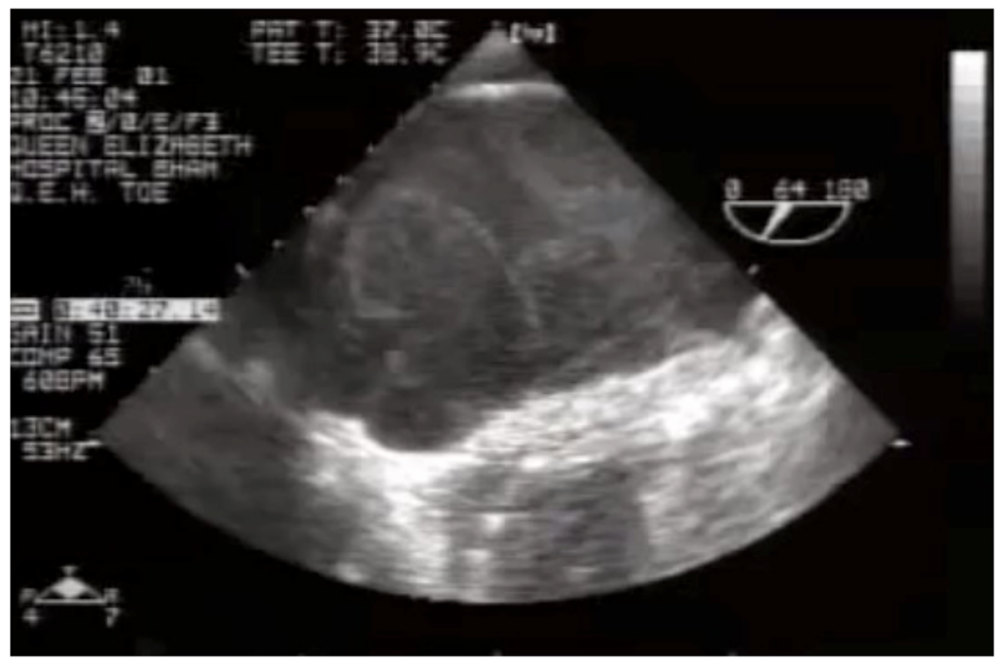

Fig. 2 Transoesophageal echocardiogram demonstrating spontaneous contrast in the Fontan circuit 
In addition it could be argued that in a serial circulation such as this, increases in pulmonary vascular resistance would be reflected by a matched increase in the ventricular end diastolic pressure and the transpulmonary gradient would change little or not at all. Therefore even the best conducted cardiac catheterisation may not allow us to demonstrate significant haemodynamic changes. In the absence of identifiable factors leading to impairment of the Fontan circulation, pulmonary vascular remodelling can be considered a potential cause of deterioration.

A number of investigators have reported improvement using pulmonary vasodilator therapy in patients with evidence of Fontan failure at various ages [6-9]. This has led to a number of groups conducting open label studies of targeted pulmonary vasodilators with varying results [10-14]. Given that endothelin receptor antagonists (ERA) have a recognised potential class effect on liver transaminase levels, there has been understandable concern on the impact ERAs may have in Fontan patients with pre-existent liver fibrosis. Recent studies have, however, demonstrated ERAs to be safe in this setting, encouraging further clinical trials [15].

The TEMPO study was a rigorously conducted randomised placebo-controlled study on the impact of the ERA bosentan on exercise capacity in adult Fontan patients. There was a small but significant increase in peak $\mathrm{VO}_{2}$ after 14 weeks of treatment, with an associated improvement in functional class $[16,17]$. However, the majority of patients included were asymptomatic, potentially limiting the study's ability to assess the impact of therapy on exercise capacity and symptoms.

It could be argued that, in the absence of pulmonary vascular remodelling, Fontan patients are unlikely to benefit from targeted pulmonary vasodilator therapy. Yet, accurately measuring pulmonary vascular resistance in Fontan patients who have a low cardiac output and who may have multiple sources of pulmonary blood flow is difficult. Earlier studies of patients undergoing cardiac transplantation for Fontan failure showed that pulmonary vascular resistance was consistently underestimated in the Fontan patient [18] and pulmonary vascular changes were present even in infants with early Fontan failure $[19,20]$. In our case, pulmonary vasodilator therapy was used successfully to treat a patient with a failing Fontan circulation but no evidence of a raised pulmonary vascular resistance, suggesting that the indications for using such therapies may go beyond invasive haemodynamics, and should be based on the overall clinical picture. In our case we presumed in the absence of other attributable causes of PLE and Fontan failure that elevation of the pulmonary vascular resistance may be contributing and warranted a trial of therapy.

Therefore, a pragmatic trial design, with exclusion criteria that remove all other likely causes of Fontan failure, was developed for the RUBATO study, for which we are currently recruiting [21]. This is a randomised placebocontrolled study assessing the efficacy of the ERA macitentan on exercise capacity in symptomatic adults and adolescents following total cavopulmonary connection Fontan surgery. The primary endpoint is change in peak oxygen consumption $\left(\mathrm{VO}_{2} \max \right)$ after 16 weeks of treatment.

At this stage, there is insufficient evidence to recommend the routine use of pulmonary vasodilator therapy in Fontan patients and we await the results of ongoing studies. However, available evidence suggests that it may be worth considering a trial of such agents in Fontan patients refractory to conventional therapy, in whom no other reversible cause has been demonstrated.

\section{Conclusions}

The Fontan circulation is dependent on systemic venous hypertension and a low pulmonary vascular resistance to function optimally. The lack of a sub-pulmonary ventricle leads to a reduced cardiac output on exercise, which patients often tolerate well, allowing survival to adult life in most cases. In the absence of identifiable causes for late Fontan failure, it may be reasonable to assume that pulmonary vascular remodelling is present and contributes to an increase in resistance within the Fontan circulation. In our case, we have highlighted the benefit of targeted pulmonary vasodilator therapy in an adult with atrio-pulmonary Fontan with symptomatic deterioration and PLE. Routine use of pulmonary vasodilators cannot be advised in the absence of robust trial evidence, but is reserved for patients with Fontan failure in whom standard therapy has failed who are under expert care.

\section{Abbreviations}

ERA: Endothelin receptor antagonist; INR: International normalized ratio; PLE: Protein losing enteropathy; TCPC: Total cavopulmonary connection; $\mathrm{VO}_{2}$ : Peak oxygen consumption

\section{Acknowledgements \\ I wish to acknowledge Dr Konstantinos Dimopoulos, Professor Robert Tulloh and Dr Robin Condliffe, who, as members of the CHAMPION steering committee, reviewed the manuscript.}

\section{About this supplement \\ This article has been published as part of Journal of Congenital Cardiology Volume 4 Supplement 1 2020: Unmet needs in Pulmonary Hypertension associated with Adult Congenital Heart Disease (ACHD-PH). The full contents of the supplement are available at https://jcongenitalcardiology. biomedcentral.com/articles/supplements/volume-4-supplement-1.}

\section{Author's contributions}

PC wrote and edited the article. The author(s) read and approved the final manuscript. 
Services (MEGS) grant and Actelion Pharmaceuticals UK Limited (who had no influence on manuscript writing).

\section{Availability of data and materials}

Not applicable.

\section{Ethics approval and consent to participate}

No ethics approval was required.

\section{Consent for publication}

Written consent was obtained for the use of anonymised images. The case histories, including the demographic characteristics of patients, were altered in order to protect the patients' identities.

\section{Competing interests}

The CHAMPION group has received an unrestricted educational grant from Actelion Pharmaceuticals. PC has received honoraria from Actelion and Bayer. There are no competing interests that have influenced the contents of the article.

Received: 12 November 2020 Accepted: 12 November 2020

Published: 16 December 2020

\section{References}

1. Kreutzer $G$, et al. An operation for the correction of tricuspid atresia. J Thorac Cardiovasc Surg. 1973;66(4):613-21.

2. Fontan F, Baudet E. Surgical repair of tricuspid atresia. Thorax. 1971;26(3): 240-8.

3. Marcelletti $C$, et al. Inferior vena cava-pulmonary artery extracardiac conduit. A new form of right heart bypass. J Thorac Cardiovasc Surg. 1990;100(2): 228-32

4. de Leval MR, et al. Total cavopulmonary connection: a logical alternative to atriopulmonary connection for complex Fontan operations. Experimental studies and early clinical experience. J Thorac Cardiovasc Surg. 1988;96(5): 682-95.

5. Wilson WM, et al. Outcomes of Patients With Hypoplastic Left Heart Syndrome Reaching Adulthood After Fontan Palliation: Multicenter Study. Circulation. 2018;137(9):978-81

6. Do $P$, et al. Successful management of plastic bronchitis in a child post Fontan: case report and literature review. Lung. 2012;190(4):463-8.

7. Haseyama K, et al. Pulmonary vasodilation therapy with sildenafil citrate in a patient with plastic bronchitis after the Fontan procedure for hypoplastic left heart syndrome. J Thorac Cardiovasc Surg. 2006;132(5):1232-3.

8. Uzun $\mathrm{O}$, et al. Resolution of protein-losing enteropathy and normalization of mesenteric Doppler flow with sildenafil after Fontan. Ann Thorac Surg. 2006; 82(6):e39-40.

9. Demetriades $\mathrm{P}$, et al. The use of Macitentan in Fontan circulation: a case report. BMC Cardiovasc Disord. 2017;17(1):131.

10. Giardini A, et al. Effect of sildenafil on haemodynamic response to exercise and exercise capacity in Fontan patients. Eur Heart J. 2008;29(13):1681-7.

11. Rhodes J, et al., Effect of inhaled iloprost on the exercise function of Fontan patients: A demonstration of concept. Int J Cardiol, 2013;168(3):2435-40.

12. Snarr BS, et al. Pulmonary vasodilator therapy in the failing Fontan circulation: rationale and efficacy. Cardiol Young. 2015;25(8):1489-92.

13. Derk $\mathrm{G}$, et al. Efficacy of endothelin blockade in adults with Fontan physiology. Congenit Heart Dis. 2015;10(1):E11-6.

14. Schuuring MJ, et al. Impact of bosentan on exercise capacity in adults after the Fontan procedure: a randomized controlled trial. Eur J Heart Fail. 2013; 15(6):690-8.

15. Bowater SE, et al. The safety and effects of bosentan in patients with a Fontan circulation. Congenit Heart Dis. 2012;7(3):243-9.

16. Hebert $A$, et al. The effect of Bosentan on exercise capacity in Fontan patients; rationale and design for the TEMPO study. BMC Cardiovasc Disord. 2013;13:36.

17. Hebert A, et al. Bosentan improves exercise capacity in adolescents and adults after Fontan operation: the TEMPO (Treatment With Endothelin Receptor Antagonist in Fontan Patients, a Randomized, Placebo-Controlled, Double-Blind Study Measuring Peak Oxygen Consumption) study. Circulation. 2014;130(23):2021-30.
18. Mitchell MB, et al. Evidence of pulmonary vascular disease after heart transplantation for Fontan circulation failure. J Thorac Cardiovasc Surg. 2004 128(5):693-702.

19. Maeda K, et al., Reevaluation of histomorphometric analysis of lung tissue in decision making for better patient selection for fontan-type operations. Ann Thorac Surg, 2004. 78(4): p. 1371-81; discussion 1371-81.

20. Levy $\mathrm{M}$, et al. Histomorphometric analysis of pulmonary vessels in single ventricle for better selection of patients for the Fontan operation. J Thorac Cardiovasc Surg. 2002;123(2):263-70.

21. Clinical Study Assessing the Efficacy and Safety of Macitentan in Fontanpalliated Subjects (RUBATO) accessed on 12 August. 2020. Available from: https://clinicaltrials.gov/ct2/show/study/NCT03153137.

\section{Publisher's Note}

Springer Nature remains neutral with regard to jurisdictional claims in published maps and institutional affiliations.
Ready to submit your research? Choose BMC and benefit from:

- fast, convenient online submission

- thorough peer review by experienced researchers in your field

- rapid publication on acceptance

- support for research data, including large and complex data types

- gold Open Access which fosters wider collaboration and increased citations

- maximum visibility for your research: over $100 \mathrm{M}$ website views per year

At BMC, research is always in progress.

Learn more biomedcentral.com/submissions 